\title{
Simulating migrated and inverted seismic data by filtering a geologic model
}

\author{
Gerrit Toxopeus ${ }^{1}$, Jan Thorbecke ${ }^{2}$, Kees Wapenaar ${ }^{2}$, Steen Petersen ${ }^{3}$, Evert Slob ${ }^{2}$, and \\ Jacob Fokkema
}

\begin{abstract}
The simulation of migrated and inverted data is hampered by the high computational cost of generating 3D synthetic data, followed by processes of migration and inversion. For example, simulating the migrated seismic signature of subtle stratigraphic traps demands the expensive exercise of 3D forward modeling, followed by 3D migration of the synthetic seismograms. This computational cost can be overcome using a strategy for simulating migrated and inverted data by filtering a geologic model with 3D spatial-resolution and angle filters, respectively. A key property of the approach is this: The geologic model that describes a target zone is decoupled from the macrovelocity model used to compute the filters. The process enables a target-oriented
\end{abstract}

approach, by which a geologically detailed earth model describing a reservoir is adjusted without having to recalculate the filters. Because a spatial-resolution filter combines the results of the modeling and migration operators, the simulated images can be compared directly to a real migration image. We decompose the spatial-resolution filter into two parts and show that applying one of those parts produces output directly comparable to 1D inverted real data. Two-dimensional synthetic examples that include seismic uncertainties demonstrate the usefulness of the approach. Results from a real data example show that horizontal smearing, which is not simulated by the 1D convolution model result, is essential to understand the seismic expression of the deformation related to sulfate dissolution and karst collapse.

\section{INTRODUCTION}

To simulate seismic images for a range of geologic models that differ only in a target zone, a convolution is commonly performed between a source signature and each reflectivity trace derived from the geologic models. This method is known as the $1 \mathrm{D}$ convolution model (Sheriff, 2001). It is computationally efficient because the 1D filter is obtained at low computational costs (White and Simm, 2003) and is reused to test the different geologic scenarios. The reflectivity traces are derived from the velocity and density logs using the Zoeppritz equations (Aki and Richards, 2002).

To properly compare the simulated and real images in the time domain, the $1 \mathrm{D}$ convolution result can be depth-to-time converted using various ray-tracing techniques. This procedure is used, for example, by Pratson and Gouveia (2002) and Braaksma et al. (2006).
A restriction is that the $1 \mathrm{D}$ convolution model is based on the assumption that the earth is horizontally layered locally. Therefore, it correctly expresses only the vertical resolution of a real migration image of horizontal layers. In addition, it does not account for the lateral resolution aspects of the migration process (Thore, 2006).

A new approach has been proposed to simulate migrated data using a combined operator that combines the result of the forwardmodeling and migration operators. We represent the combined operator with a spatial-resolution filter, also known as point-spread function or PSF (Devaney, 1984; Gjøystdal et al., 2002), Green's functions for migration (Schuster and $\mathrm{Hu}, 2000$ ), resolution function (Gelius et al., 2002), and illumination-response function (Xie et al., 2006). A migration image is simulated by filtering a model with a $3 \mathrm{D}$ spatial-resolution filter (Schuster and $\mathrm{Hu}, 2000$; Lecomte et al., 2003). This new approach has three advantages. First, the spatial-

Manuscript received by the Editor 5 September 2006; revised manuscript received 5 September 2007; published online 18 January 2008

${ }^{1}$ Formerly Delft University of Technology; presently StatoilHydro, Oslo, Norway. E-mail: GTox@ StatoilHydro.com.

${ }^{2}$ Delft University of Technology, Department of Geotechnology, Delft, The Netherlands. E-mail: J.W.thorbecke@tudelft.nl; C.P.A.Wapenaar@tudelft.nl; E.C.Slob@tudelft.nl; J.T.Fokkema@tudelft.nl.

${ }^{3}$ StatoilHydro Research Center, Bergen, Norway. E-mail: SteAP@ StatoilHydro.com.

(C) 2008 Society of Exploration Geophysicists. All rights reserved. 
-resolution filter can be constructed using Green's functions that were used to migrate the real data. Under the assumption that the overburden model is not changed, the filter can be kept constant and reused (Santos et al., 2000). Thus, we achieve the speedup required to enable iterative testing of different geologic scenarios. Second, the spatial-resolution filter combines the result of the forward modeling and migration operators. Therefore, the simulated migration image can be compared directly to the real migration image. Third, a model describing a reservoir can be geologically more detailed than the macromigration velocity model used to compute the spatial-resolution filter because the filter can be interpolated to a finer grid discretization.

In addition to the use of seismic data, geologic interpretation is also frequently constrained by estimated attributes such as acoustic impedance obtained from the seismic data by inversion procedures. Current industry practice is to estimate attributes such as acoustic impedance from the migrated real data by a seismic inversion process known as constrained sparse-spike inversion (see Veeken and Da Silva, 2004). Basically, a simulated seismic trace is created with the help of the 1D convolution model and matched to one trace of the migrated real data by varying the position and strength of a number of spikes (Oldenburg et al., 1983; van Riel and Berkhout, 1985). We refer to the final result of a seismic inversion process as a 1D inversion image. Obviously, this method is limited by the $1 \mathrm{D}$ assumption.

Our goal is to test different geologic scenarios of a target zone by filtering a model with filters that simulate migration as well as impedance inversion images. First, we discuss computing a migration image conceptually. Next, we decompose a spatial-resolution filter into two new filters. Third, we analyze a synthetic example of dipping reflectors and show a case study. The discussion also addresses how to include seismic uncertainties. Finally, the computation of an impedance inversion image is discussed conceptually, and a synthetic example of simulating a 1D inversion image is shown.

Though the $2 \mathrm{D}$ examples presented show only P-wave data, the approach is valid in three dimensions for an arbitrary elastic earth model. In the case study we present, the variations in the strike direction are likely to be small compared to those in the dip direction. Therefore, 3D migrated and inverted data are thought to be well approximated by migrated and inverted 2D cross sections.

\section{SIMULATING MIGRATED SEISMIC DATA BY FILTERING A MODEL}

Acquired seismic data $d$ can be described as

$$
d\left(\mathbf{x}_{D}, \mathbf{x}_{S}, t\right)=L m,
$$

where $\mathbf{x}_{D}$ and $\mathbf{x}_{S}$ denote the spatial coordinate vectors of the detector and source positions, respectively, and $t$ denotes time. The seismic experiment is denoted by $L$ and the earth by $m$.

Similarly, simulated seismic data $\hat{d}$ are obtained by a forward operator $\mathcal{L}$ that acts on a detailed model $\hat{m}$. The model is a possible representation of the earth in terms of the parameters dominating the seismic measurements. The forward operator models the real seismic experiment (Figure 1), according to

$$
\hat{d}\left(\mathbf{x}_{D}, \mathbf{x}_{S}, t\right)=\mathcal{L} \hat{m}(\mathbf{x}),
$$

where $\mathbf{x}$ denotes the spatial coordinate vector of position in the earth model. From the seismic measurements, a structural image of the earth is obtained by using a migration operator $\mathcal{M}$. The operator $\mathcal{M}$ is the approximate inverse of $\mathcal{L}$ in some sense (for example, the adjoint [Schuster and $\mathrm{Hu}, 2000])$. The same migration operator can be used to image the simulated seismic data. This extends the relations to

$$
\begin{aligned}
& m_{\text {mig }}(\mathbf{x})=\mathcal{M d}\left(\mathbf{x}_{D}, \mathbf{x}_{S}, t\right), \\
& \hat{m}_{\text {mig }}(\mathbf{x})=\mathcal{M} \hat{d}\left(\mathbf{x}_{D}, \mathbf{x}_{S}, t\right),
\end{aligned}
$$

where $m_{\text {mig }}$ and $\hat{m}_{\text {mig }}$ denote the real and simulated migration image, respectively. The operator $\mathcal{M}$ can represent full prestack migration, common-offset migration $\left(\mathbf{x}_{D}-\mathbf{x}_{S}=\right.$ constant $)$, or zero-offset migration $\left(\mathbf{x}_{D}=\mathbf{x}_{S}\right)$.

A seismic interpreter is concerned with how and to what extent geologic details (in $\mathrm{m}$ ) are visible in the migration image $m_{\text {mig. }}$. Ideally, the interpreter should investigate the following relation:

$$
\hat{m}_{\text {mig }}(\mathbf{x})=\mathcal{M}\{\mathcal{L} \hat{m}(\mathbf{x})\}
$$

By comparing $\hat{m}_{\text {mig }}(\mathbf{x})$ with $m_{\text {mig }}(\mathbf{x})$, the interpreter aims to validate $\hat{m}(\mathbf{x})$ as a representation of $m$. Equation 4 combines the operations of this total process (illustrated by the second column in Figure

1). Therefore, to simulate migrated data a combined operator $(\mathcal{C})$ is introduced to represent the two operators

$$
\hat{m}_{\text {mig }}(\mathbf{x})=\{\mathcal{M L}\} \hat{m}(\mathbf{x})=\mathcal{C} \hat{m}(\mathbf{x}),
$$

where $\mathcal{C}=\mathcal{M L}$.

\section{Application I: Simulating migrated seismic data}

The combined operator is represented by a spatial-resolution filter $C$, which is obtained by computing the image of a single unit-strength scatterer in a macrovelocity model (the impulse response of the filter). Using the spatial-resolution filter, we simulate migrated data as
Figure 1. Summary of how prestack depth-migrated data are simulated by filtering an earth model with a spatial-resolution filter. The spatial-resolution filter is decomposed into an angle and band-limitation filter. The macrovelocity model (interval velocities) used to migrate the real measurements computes the spatial-resolution filter. 


$$
\hat{m}_{\text {mig }}(\mathbf{x})=\mathcal{C} \hat{m}(\mathbf{x})=\int C\left(\mathbf{x}, \mathbf{x}^{\prime}\right) \hat{m}\left(\mathbf{x}^{\prime}\right) d \mathbf{x}^{\prime},
$$

where

$$
C\left(\mathbf{x}, \mathbf{x}^{\prime}\right)=\mathcal{C} \delta\left(\mathbf{x}-\mathbf{x}^{\prime}\right),
$$

with $\mathbf{x}^{\prime}$ the spatial coordinate vector of the unit-strength scatterer. Thus, instead of forward-modeling shot records and subsequently applying a migration algorithm, an earth model is filtered by a spatial-resolution filter to simulate migrated data (illustrated by the third column in Figure 1). The spatial-resolution filter resembles the processes of the real measurement and the migration algorithm. Therefore, the simulated migrated data can be compared directly to the migrated real data.

In the remainder of this article, we use operator notation but refer to the operator as a filter because we filter an earth model to simulate migrated data. Ideally, to simulate a migration image, a spatial-resolution filter is computed for each subsurface point in the target zone. However, this process is too expensive computationally, and interpolating a spatial-resolution filter by averaging two neighboring spatial-resolution filters is not easy because the shape of the filter can have a large degree of lateral variation. In common practice, limited numbers of spatial-resolution filters are computed and are assumed to be constant around the scatterer positions $\mathbf{x}^{\prime}$. This computation procedure is used, for example, by Lecomte et al. (2003) and in this article.

\section{Decomposing the spatial-resolution filter}

Now we show that the spatial-resolution filter can be decomposed into two new filters. These filters are named an angle $\mathcal{A}$ and a bandlimitation $\mathcal{B}$ filter. Using these two filters, a simulated migrated seismic image is approximated by

$$
\hat{m}_{\text {mig }}(\mathbf{x})=\underbrace{\mathcal{B} \mathcal{A}}_{C} \hat{m}(\mathbf{x})
$$

In a model with a constant P-wave velocity of $2000 \mathrm{~m} / \mathrm{s}$, a single unit-strength scatterer is buried at a depth of $2000 \mathrm{~m}$. Symmetrically overlying this scatterer is a zero-offset acquisition setup, with a total recording aperture of $3000 \mathrm{~m}$ (Figure 2a). The source signature is a Ricker wavelet with central frequency of $32 \mathrm{~Hz}$, filtered with a bandpass filter of 5-70 $\mathrm{Hz}$ and sampled at $4 \mathrm{~ms}$. We use equation 7 with $\mathcal{C}=\mathcal{M L}$ to compute the spatial-resolution filter. As an intermediate result, the two-way time (twt) recorded zero-offset response $\hat{d}$ is shown in Figure 2b. After migration, a spatial-resolution filter $C$ is obtained (Figure 2c). Both results are transformed to the wavenumber domain, where the $\omega$ axis of Figure 2d is scaled with half the velocity to illustrate the principle of migration: mapping one line of constant $\omega$ of Figure 2d onto one circle segment of Figure 2e (Stolt, 1978).

In the wavenumber domain, two key features of the spatial-resolution filters are observed. First, the spectrum is limited from $\omega_{\min }$ $=10 \pi$ to $\omega_{\max }=140 \pi(\mathrm{rad} / \mathrm{s})$, where $\omega=2 \pi f(f$ denoting the frequency in hertz). Second, the spatial-resolution filter is constrained by the minimum and maximum angles of wave propagation $\left(\varphi_{1}\right.$ and $\left.\varphi_{2}\right)$. At $\pm 90^{\circ}$, these angles mark the boundary between the propagating and evanescent parts of a wavefield (Berkhout, 1987; Wapenaar and Berkhout, 1989). In practice, these angles are less than $90^{\circ}$ because of a limited recording aperture and overburden ef- fects. In a homogeneous medium, these angles are directly related to the total recording aperture (Schuster and $\mathrm{Hu}, 2000$ ). In this example, the background medium is homogeneous and the acquisition setup is symmetrically overlying the scatterer, so the minimum and maximum angles of wave propagation are symmetrical (i.e., $-\varphi_{1}$ $\left.=\varphi_{2}\right)$.

Now that the two key features are identified, an alternative way of obtaining a spatial-resolution filter can be developed by directly constructing two new filters in the wavenumber domain. The first filter, an angle filter, uses the minimum and maximum angles of wave propagation to form a stop/pass filter (Figure 3a). These angles can be obtained from ray tracing, a local plane-wave analysis, or the filter computed from the combined operator (equation 7) if a delta function is used as source signature.

The second filter is named a band-limitation filter. It is circular and contains the spectrum of the wavelet along its radius (Figure $3 \mathrm{~b}$ ). Inverse Fourier transformation shows the two filters in the space domain (Figure 3d and e). Multiplication of the two filters (in the wavenumber domain) and subsequent inverse Fourier transformation give a spatial-resolution filter, which is the same as that obtained from the combined operator (compare Figures $2 \mathrm{c}$ and $3 \mathrm{f}$ ). This shows that the spatial-resolution filter $\mathcal{C}$ can be interpreted as acting as two separate filters, summarized as $\mathcal{C}=\mathcal{B} \mathcal{A}$, where $\mathcal{A}$ is the angle filter and $\mathcal{B}$ is the band-limitation filter.

\section{CALCULATING SPATIAL-RESOLUTION FILTERS}

Three approaches to calculating spatial-resolution filters are found in the literature: (1) closed-form expression, (2), angle and band-limitation filter, and (3) combined operator (equation 7). The first approach assumes a homogeneous velocity model; Chen and Schuster (1999) present a closed-form expression for the zero-offset case. Input to the second approach is directional information derived using ray tracing (Lecomte and Gelius, 1998) or obtained from a one-way wavefield by a local plane-wave analysis (Xie et al., 2006).

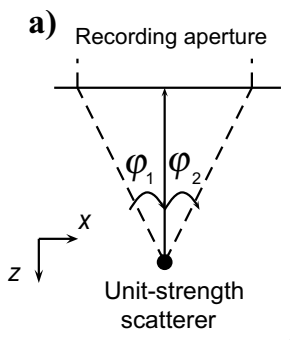

d)

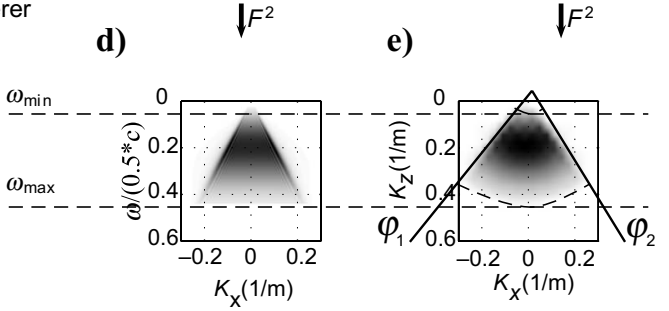

Figure 2. (a) An acquisition setup with a recording aperture of $3000 \mathrm{~m}$ overlies a single unit-strength scatterer at $2000 \mathrm{~m}$ depth. (b) The forward-modeled response from the scatterer. (c) A spatial-resolution filter obtained by migrating the response of Figure $2 \mathrm{~b}$. (d, e) Fourier-transformed results of (b) and (c). $F^{2}$ denotes the forward 2D spatial Fourier transform. The minimum and maximum angles of wave propagation are denoted by $\varphi_{1}$ and $\varphi_{2}$, and the spectrum is limited by $\omega_{\min }$ and $\omega_{\max }$. 
In the third approach, computational savings can be made by reusing the Green's functions of the migration process applied to the real data. In addition, the directional information can be used to compute the reflection model properly (Gjøystdal et al., 2007).

In this article, the third approach is used to calculate the spatialresolution filters. The main reason is that the approach allows us to study the effect of using a different background velocity model than the one used to migrate the real data. Currently, in the second approach, traveltime differences cannot be used. Traveltimes are used only as additional information for weighting the filters with respect to amplitudes.

Three steps summarize a numerical recipe of the third approach. In the first step, the one-way response of a unit-strength scatterer is computed with the aid of an exploding reflector model (Loewenthal et al., 1976; Yilmaz, 2001). We calculate the Green's functions with one-way wave-equation-based operators as described by Thorbecke et al. (2004). In the second step, a two-way common-offset data set is created by convolving the Green's functions, corresponding to the proper source and receiver paths given a specific offset (Deregowski and Rocca, 1981). Finally, a common-offset spatial-resolution filter is computed by applying a common-offset migration algorithm to the common-offset data (equation 7 with $\mathcal{C}=\mathcal{M L}$ ). Because information on the incidence angle is needed to compute the reflectivity grid properly, additional ray tracing is needed.

It is important to understand that all acquisition and model heterogeneity-related effects can be captured properly in a spatial-resolution filter. As an initial approach, we simulate primary reflection-migrated data and compare only the phase of the simulated and reference data. Therefore, in the comparison, we tacitly assume that all multiple scattering events have been properly removed from the data or imaged to their correct position of origin. Removing multiple energy is of great importance in seismic processing.

Current industry practice is to remove the free surface scattering from the real data; internal multiple scattering is only partly removed (Verschuur et al., 1992; Hill et al., 1999; Matson and Dragoset, 2005). To capture the effects of internal multiple scattering in a spa-

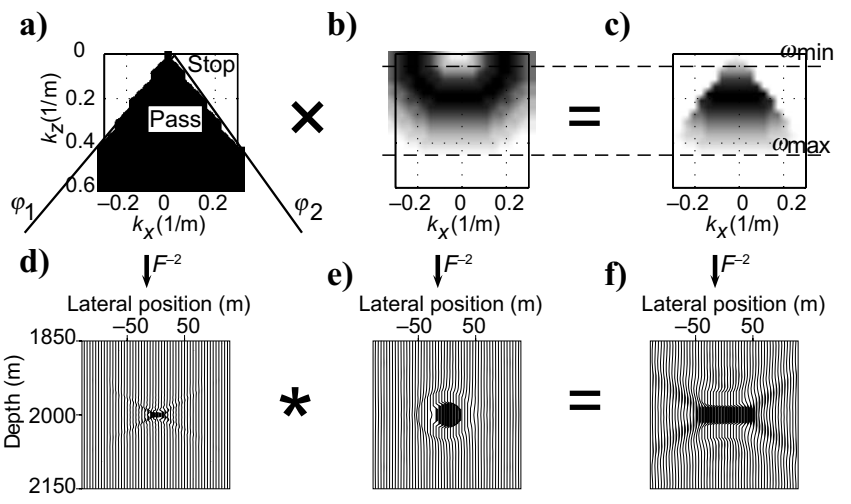

Figure 3. (a) An angle filter in the wavenumber domain. (b) A bandlimitation filter in the wavenumber domain. (c) A spatial-resolution filter in the wavenumber domain. (d) An angle filter in the space domain. (e) A band-limitation filter in the space domain. (f) A spatialresolution filter in the space domain. The term $F^{-2}$ denotes the inverse $2 \mathrm{D}$ spatial Fourier transform. A multiplication and multidimensional spatial convolution are denoted by $\times$ and $*$, respectively. The minimum and maximum angles of wave propagation are denoted by $\varphi_{1}$ and $\varphi_{2}$. The spectrum is limited by $\omega_{\min }$ and $\omega_{\max }$, which resembles the spectrum of the source signature in the real measurements. tial-resolution filter, the recipe of the third approach would change as follows. The one-way operators used in the first step are replaced by a two-way finite-difference operator. Amplitude effects of a real migration image can be taken into account in a similar manner. For example, anelastic and/or transmission losses can be simulated by including these effects in the forward-modeling algorithm and neglecting them in depth migration. This conforms to the industry practice for migration where these effects are not taken into account.

\section{INFLUENCE OF A SPATIAL-RESOLUTION FILTER ON A REFLECTIVITY MODEL}

The model we use starts at a depth of $2550 \mathrm{~m}$ and consists of five interfaces, embedded in a homogeneous background. The background P-wave velocity is $2000 \mathrm{~m} / \mathrm{s}$. The source is a Ricker wavelet with a peak frequency of $31 \mathrm{~Hz}$. The interfaces have dips of $0^{\circ}, 30^{\circ}$, $45^{\circ}, 60^{\circ}$, and $75^{\circ}$, with corresponding normal-incidence reflection strengths of $0.05,0.03,-0.04,0.07$, and 0.06 , respectively (Figure 4). The horizontal interface contains two gaps of 8 and $20 \mathrm{~m}$. Obviously, these gaps may not exist in a real geologic model, but they are included to illustrate horizontal smearing in a migration image.

First, we discuss the 1D convolution method. We convert the Ricker wavelet to depth using half of the background velocity (Figure 5a). We then simulate a seismic image by convolving the wavelet with each trace of the reflectivity model of Figure 4. By displaying the successive seismic traces, the $1 \mathrm{D}$ convolution model result is obtained (Figure 5b).

Next, a spatial-resolution filter is obtained using the same Ricker wavelet. The total recording aperture is chosen such that the minimum and maximum angles of wave propagation are $\pm 70^{\circ}$ (Figure $6 a)$. A migration image is simulated by performing a multidimensional spatial convolution between the spatial-resolution filter and the reflectivity model (Figure 4). The result is shown in Figure 6b.

In the following discussion, three aspects of the two simulated seismic images of Figures $5 \mathrm{~b}$ and $6 \mathrm{~b}$ are compared: suppression and blurring of geological reflectors, migration wavelet stretch, and horizontal smearing. First, when we compare the seismic events in Figures 4 and $6 \mathrm{~b}$, observe that the $75^{\circ}$ dip is not imaged in Figure $6 \mathrm{~b}$. This is directly related to the minimum and maximum angles of wave propagation. A spatial-resolution filter suppresses geological dips that lie outside its range of angles of wave propagation, similar

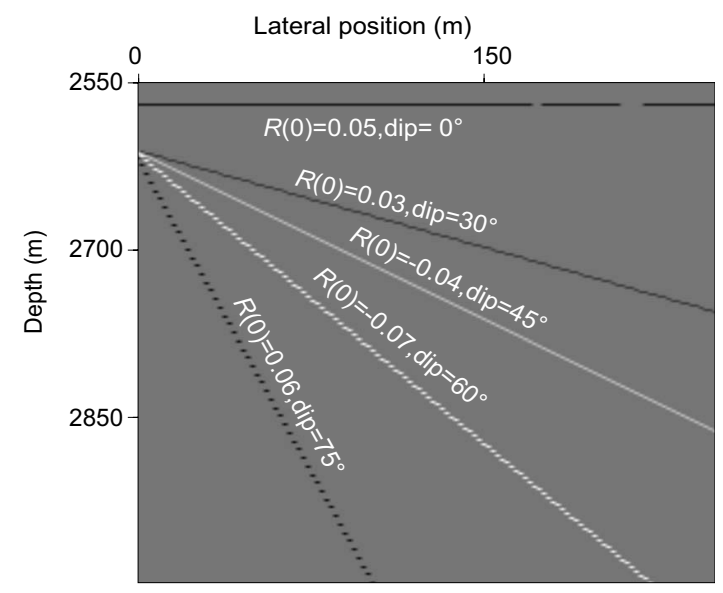

Figure 4. A normal-incidence reflectivity model embedded in a homogeneous background model with a P-wave velocity of $2000 \mathrm{~m} / \mathrm{s}$. 
to migration (e.g., Beylkin and Burridge, 1984; Bleistein et al., 2000, chapter 4; Lecomte et al., 2003).

Second, from the three different seismic images, a trace at a lateral position of $140 \mathrm{~m}$ is selected (Figure 7). The peak and trough values correspond to the reflectivity values. However, with increasing geologic dip angle, the wavelets of the simulated migration images are stretched. This feature also occurs in real data migration and is known as migration wavelet stretch (Wapenaar et al., 1999). The stretch amounts to $1 / \cos$ (angle of geologic dip). Thus, for a horizontal layer, filtering by a wavelet or a spatial-resolution filter results in the same wavelet. However, at a $60^{\circ}$ geologic dip, the wavelet resulting from filtering the reflectivity model with the spatial-resolution filter results in a wavelet that is twice as long (as illustrated by two overlapping arrows in Figure 7).

Finally, the influence of the two gaps of 8 and $20 \mathrm{~m}$ in the reflectivity model are investigated by considering a horizontal slice at $2575 \mathrm{~m}$ through the Figures 4, 5b, and 6b. The simulated images show the same amplitude value (away from the gaps and edge).

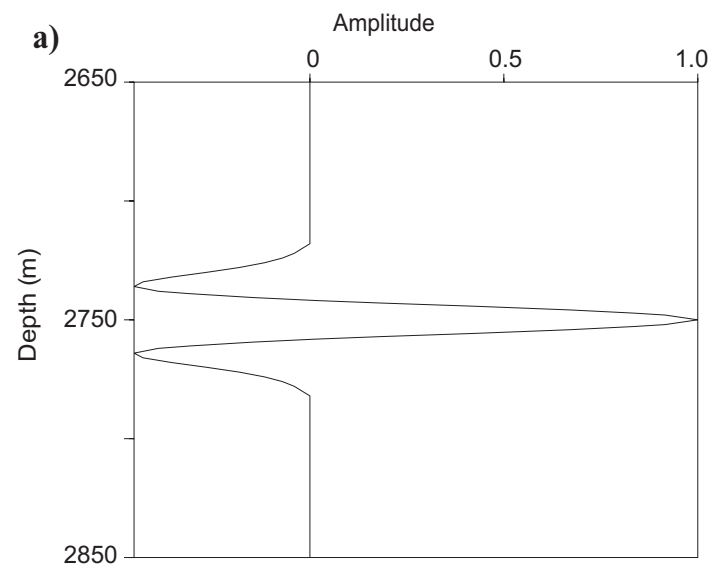

b) Lateral position $(m)$

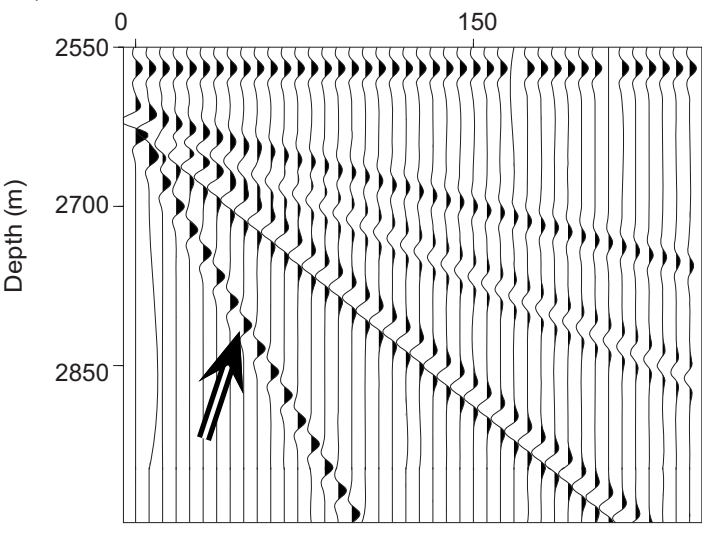

Figure 5. (a) A 1D wavelet. (b) The 1D convolution model result, obtained by convolving each trace of the normal-incidence reflectivity model with the 1D wavelet (Figure 4 and Figure 5a). The arrow indicates the position of the $75^{\circ}$ reflector.
However, around the gaps, their responses differ (Figure 8). The 1D convolution method result represents the values of the reflectivity model. The $70^{\circ}$ spatial-resolution filter has almost smeared the $8-\mathrm{m}$ gap, similar to what would happen in migration.

The above result agrees with the theoretically computed horizontal resolution of a migration image given by $\lambda /\left[2\left(\sin \varphi_{2}-\sin \varphi_{1}\right)\right]$ (Claerbout, 1976; Chen and Schuster, 1999). Here, $\lambda=c / f_{\max }$, with $c=2000 \mathrm{~m} / \mathrm{s}$ and $f_{\max }=70 \mathrm{~Hz} ; \varphi_{1}$ and $\varphi_{2}$ are the minimum and maximum angles of wave propagation, respectively. For $-\varphi_{1}=\varphi_{2}$ $=70^{\circ}$, this results in a horizontal resolution of approximately $8 \mathrm{~m}$. The relation shows that the horizontal resolution depends on the angles of wave propagation. As a result, for a wide aperture, the gap is imaged (Figure 6b); for a small aperture, the gap will be smeared. The vertical resolution is quantified by $\lambda / 4$ (Kallweit and Wood, 1982; Vermeer, 2001), which is less than the horizontal resolution.

Lecomte (2006) presents a similar analysis by analyzing simulated and reference images in the wavenumber domain.
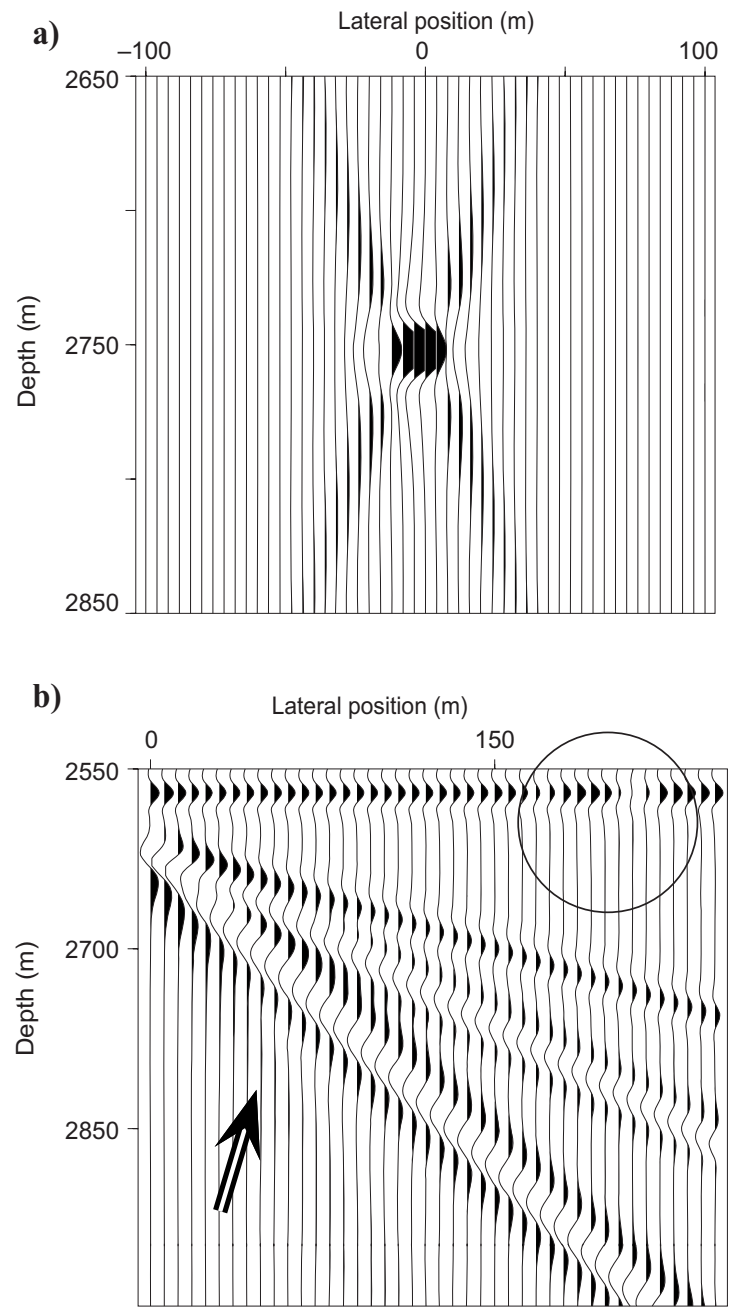

Figure 6. (a) A $70^{\circ}$ spatial-resolution filter. (b) A simulated migration image, obtained by convolving the normal-incidence reflectivity model with the spatial-resolution filter (Figures 4 and 6a). The arrow indicates the position of the $75^{\circ}$ reflector. The circle highlights a difference in horizontal resolution. 


\section{SEISMIC MODELING OF SULFATE DISSOLUTION AND KARST COLLAPSE-RELATED DEFORMATION}

The purpose of this section is to understand the sulfate dissolution and karst collapse-related deformation. Over the crests of a field, the structure of a reservoir is complicated by the dissolution of a sulfate layer located 100-140 m below the reservoir. Figure 9 shows an inline section of the 3D migrated data of this area. We refer to this image as the reference.

The geologic model is based on interpretation of 3D seismic data, well logs, cores, and outcrop analogs. The model consists of various compartments representing different properties. Each is populated with rock properties derived from wells. The earth model is discretized at depth and offset with step sizes of $d z=1.5 \mathrm{~m}$ and $d x$ $=13 \mathrm{~m}$. As discussed, the spatial-resolution filter is constrained by

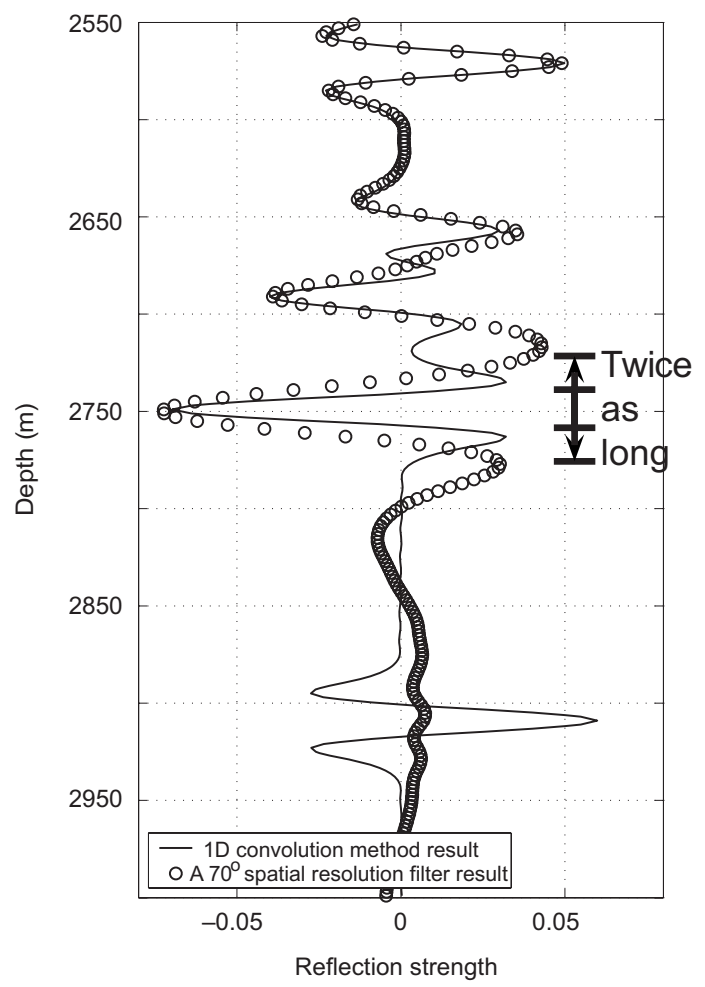

Figure 7. A trace comparison at lateral position $140 \mathrm{~m}$ of Figures $5 \mathrm{~b}$ and $6 \mathrm{~b}$. The two arrows highlight that the 1D convolution model does not simulate migration wavelet stretch.

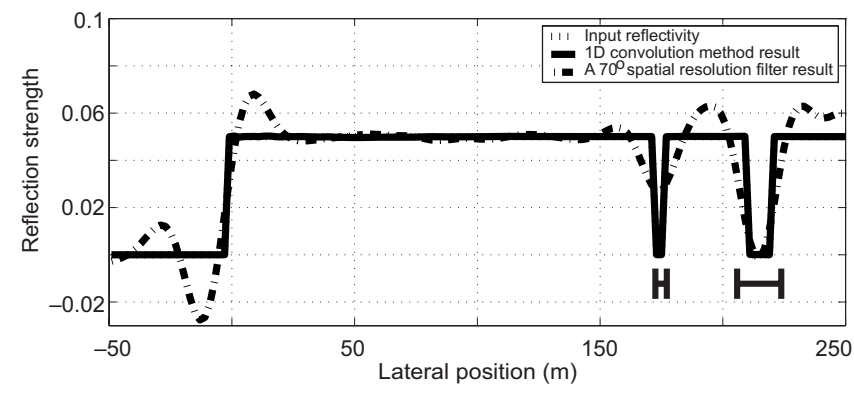

Figure 8. A depth slice through the horizontal reflector of Figures 4, $5 \mathrm{~b}$, and $6 \mathrm{~b}$. The gaps illustrate horizontal smearing. the acquisition setup, the macromigration velocity model that is used to migrate the real data, and the processing parameters of the real data. Unfortunately, for this particular data set, the migration velocity model and the processing flow are not available. As a practical approach, a total opening angle of $40^{\circ}$ is chosen $\left(-\varphi_{1}=\varphi_{2}=20^{\circ}\right)$. The other selected parameters are a Ricker wavelet with a center frequency of $13 \mathrm{~Hz}$ and discretization chosen at $d z=1 \mathrm{~m}$ and $d x$ $=13 \mathrm{~m}$.

Several iterations of the geologic model were realized and refined by comparing the phase of the simulated migrated and real migrated seismic data. The spatial-resolution filter was reused to simulate each of the different geologic scenarios. Subsequently, a linear depth-to-time conversion was used to simulate a time-migration image. (For information on the geologic scenarios and the model builder, see Jensen et al. [2004]. For information on the fluid-substitution procedure, see Petersen et al. [2003].) Figure 10 shows the geologic scenario that had the best simultaneous match of all tested scenarios, for both the input data of the model and the seismic reference data. Figure 11 shows the corresponding simulated migrated image (compare with Figure 9).

The 1D convolution model result is shown in Figure 12 for comparison with the reference data. The comparison shows that in the 1D convolution result, the expression of the deformation related to sul-

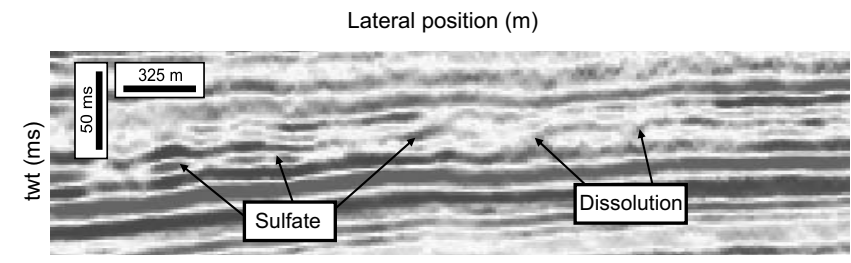

Figure 9. A prestack time-migration image. The arrows show the interpreted locations of sulfate dissolution and karst collapse.

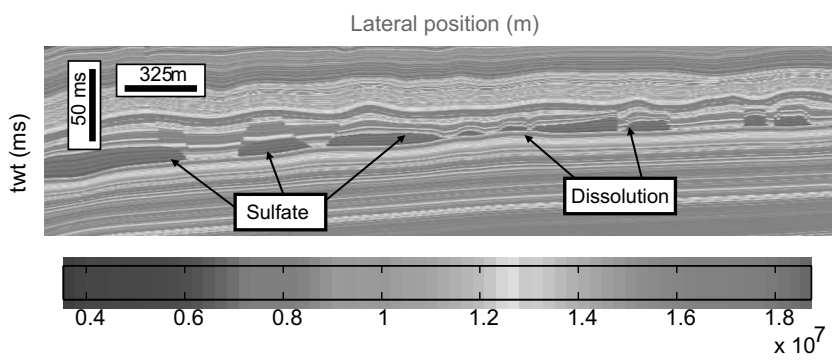

Figure 10. An impedance model. The arrows show the interpreted locations of sulfate dissolution and karst collapse. Acoustic impedance is in kilograms per square-meter-seconds $\left[\mathrm{kg} /\left(\mathrm{m}^{2} \mathrm{~s}\right)\right]$.

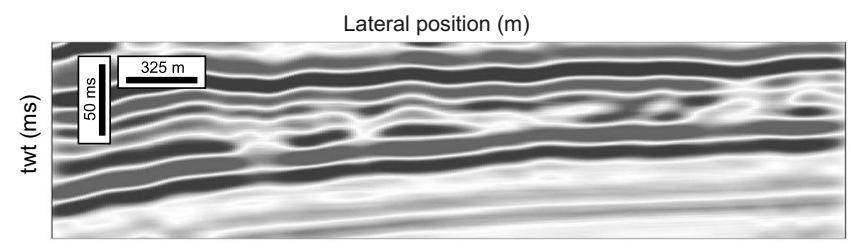

Figure 11. A simulated time-migration image obtained by filtering the normal-incidence model of Figure 10 with the $40^{\circ}$ spatial-resolution filter (not shown) followed by a linear depth-to-time conversion. 
fate dissolution and karst collapse in the seismic data is not well represented. This is because in the 1D convolution model, horizontal blurring is not taken into account. We conclude that the spatial-resolution filter is essential to simulate a migration image properly. Thematch can be improved further by including more information of the processing steps into the spatial-resolution filter, e.g., the Green's functions used to migrate the real data and $3 \mathrm{D}$ effects.

\section{Simulating a defocused migration image}

The quality of a migration image depends on seismic processing, the migration algorithm, and the velocity model, among other factors. A macrobackground velocity model needs to be derived to migrate the real measurements. When the background velocity model does not match the real earth to some degree, structural information is not imaged properly. We refer to this as a defocused migration image.

In the previous examples, the simulated results used the same macrovelocity model for both forward modeling and migration, resulting in a simulated focused migration image. If a focused simulated migration image is compared with a real defocused migration image, the model describing the target zone may be updated such that, for example, a wrong geologic dip is used to obtain the best match. Independent information is needed to reveal that the background migration velocity model is not sufficiently accurate. If this information is present, the spatial-resolution filter can be used to capture this effect instead of remigrating the real data.

First, we simulate a reference migration image from a half-graben model (Figure 13a, inspired by Mittet et al., 1995). Marine data from this model are obtained with a 2D finite-difference time-domain code using an 800-m-long detector cable (black arrow, Figure 13a) and a detector and source spacing of $10 \mathrm{~m}$ each. The first source position is $810 \mathrm{~m}$, and the last source position is $2000 \mathrm{~m}$. The source is a 25-Hz Ricker wavelet, and a sampling rate of $4 \mathrm{~ms}$ is used.

An image is obtained from prestack depth migration on the shot records. The image is defocused because, in the velocity model, the velocity of two layers is lowered, as indicated by $c_{p_{\text {migration }}}$ in Figure 13a. This produces a reference defocused migration image. The reference migration image for the zone of interest is outlined by the dashed box in Figure 13a and shown in Figure 13b. In the target zone, the wedge structure is gradually shifted upward from $x=1600 \mathrm{~m}$ to $x=1000 \mathrm{~m}$, and the entire structure is shifted laterally (highlighted by the lines in Figure 13b).

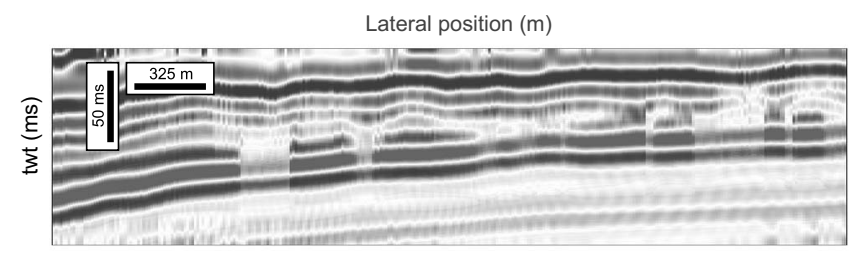

Figure 12. The 1D convolution model result obtained by filtering the normal-incidence model of Figure 10 with a $1 \mathrm{D}$ wavelet (not shown) followed by a linear depth-to-time conversion.
Finally, we compute the zero-offset forward modeling response with the modeling velocity model of nine evenly distributed diffraction points located at $x=1100 \mathrm{~m}, z=1100 \mathrm{~m}$ to $x=1500 \mathrm{~m}, z$ $=1500 \mathrm{~m}$. These data are migrated with the same migration algorithm and modified velocity model as the reference data. The spatialresolution filters are shown in Figure 13c. A rectangular grid overlying the spatial-resolution filters shows they are positioned erroneously in both the horizontal and vertical directions (Figure 13c). These are known as smiles and frowns (Zhu et al., 1998). A normalincidence reflectivity grid is derived from the modeling velocity and density models. A defocused migration image is simulated by merging the results of spatial convolutions between the nine defocused spatial-resolution filters with an equal area of the reflectivity model (Figure 13d).

The result shows that the shifting of the wedge structure is simulated and the target area is incorrectly positioned laterally (Figure $13 \mathrm{~b}$ and $\mathrm{d})$. The abrupt steps illustrate the local behavior of the spatial-resolution filter. When more spatial-resolution filters are used, a smoother image is obtained. In addition, ray-tracing information could have been used to investigate the degree of lateral variation of the shape of the spatial-resolution filter. More information can be found in Lecomte (2006). This approach may be used to understand and visualize the uncertainty of migrated data (Sambridge et al., 2006).
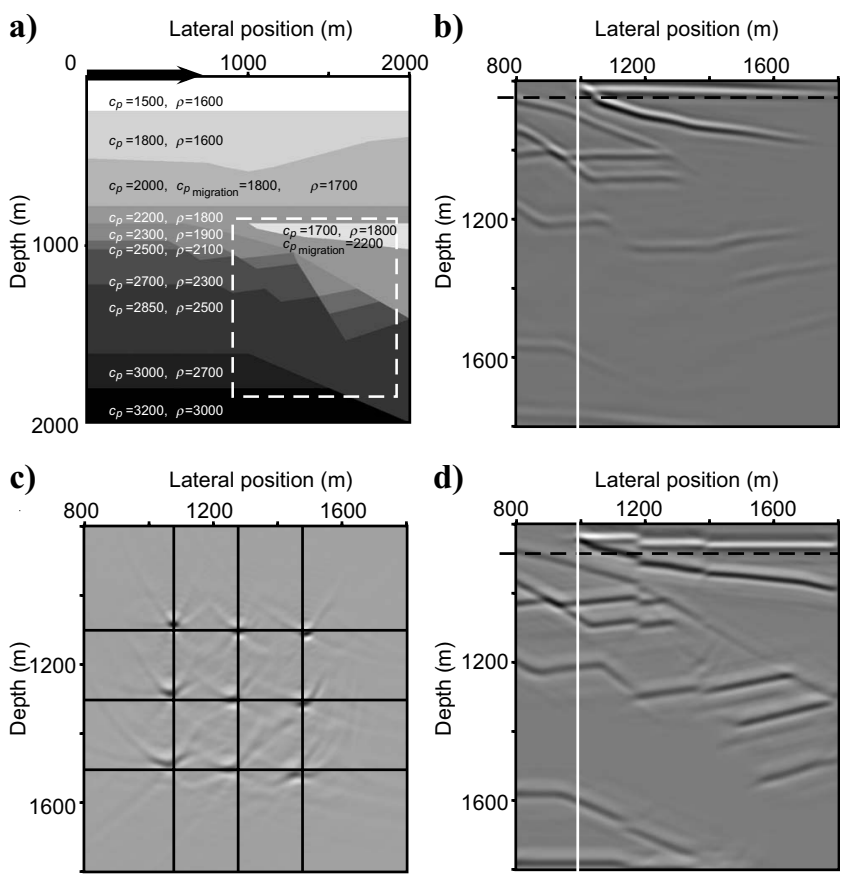

Figure 13. (a) A half-graben model. The $\mathrm{P}$-wave velocity values are in meters/second, and rock density is kilograms/cubic meter. The value $c_{p_{\text {migration }}}$ indicates the background migration velocity model differs from the modeling velocity model. The black arrow indicates the direction and length of the moving array that simulates the marine acquisition. The dashed box highlights the selected target-zone. (b) A defocused reference migration image of the target zone. (c) Nine defocused spatial-resolution filters. The grid illustrates that all nine spatial-resolution filters are defocused. (d) The simulated defocused reference migration image of the target zone. The dashed and white lines highlight the suboptimal focusing of reflectors. 


\section{SIMULATING INVERTED DATA BY FILTERING A MODEL}

The current industry practice of estimating acoustic impedance data takes migrated seismic data as input (Veeken and Da Silva, 2004), using the migrated seismic data as a starting point. The real impedance image $m_{\mathrm{inv}}$ is obtained by using a seismic inversion algorithm $\mathcal{S}$ as

$$
m_{\text {inv }}(\mathbf{x})=\mathcal{S} m_{\text {mig }}(\mathbf{x})
$$

where ideally $\mathcal{S}=\mathcal{C}^{-1}$. Similarly, a simulated impedance image is obtained by using the seismic inversion algorithm on the simulated migrated data as

$$
\hat{m}_{\text {inv }}(\mathbf{x})=\mathcal{S} \hat{m}_{\text {mig }}(\mathbf{x})=\mathcal{S}\{\mathcal{C} \hat{m}(\mathbf{x})\}=\mathcal{S}\{\mathcal{B} \mathcal{A} \hat{m}(\mathbf{x})\}
$$

Based on the literature, seismic inversion algorithms can be grouped roughly into two classes. The first class of seismic inversion algorithms attempts to remove both the angle and band-limitation filters of the migrated seismic data in the inversion process. In particular, it deals with limited acquisition aperture (see Nemeth et al., 1999). Migration-deconvolution uses spatial-resolution filters to invert the real migrated data (Yu et al., 2006).

The second class is known as constrained sparse-spike inversion and represents current industry practice. Algorithms that fall into this class use the 1D convolution model (Oldenburg et al., 1983; van Riel and Berkhout, 1985; Lancaster and Whitcombe, 2000). Hence, these algorithms do not account for the lateral resolution aspects of the migration process. In the remainder of this article, the second class of inversion algorithms is denoted as 1D inversion. The integration and scaling steps that are part of the current seismic inversion

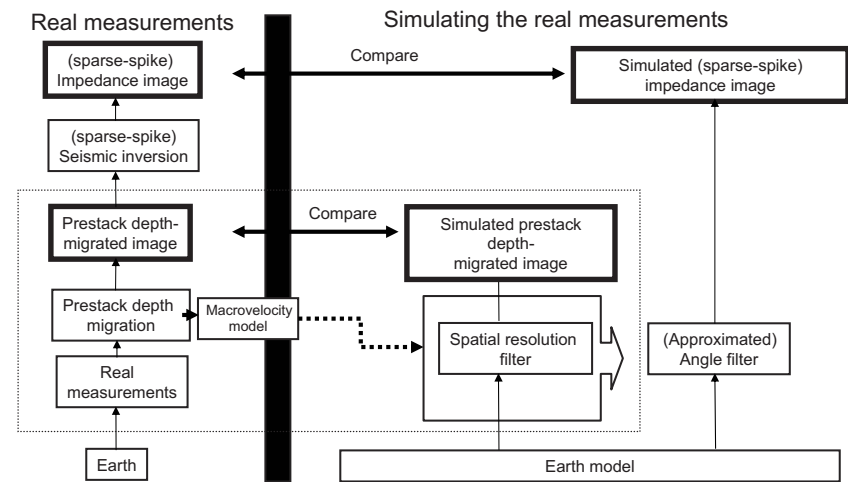

Figure 14. Simulating in a complete sense (modified from Petersen, 1992). The figure summarizes conceptually how different operators and filters are used to simulate migrated and impedance data that can be compared directly to the real data. The macrovelocity model (interval velocities) used to migrate the real measurements computes the spatial-resolution and (approximated) angle filters. The dotted box summarizes Figure 1. process are omitted for notational convenience. More details on these two steps are found in Ferguson and Margrave (1996).

Next, we show how each class removes the band-limitation and angle filters of the real migrated data.

\section{Simulating inverted data}

The first class of seismic inversion algorithms handles all aspects of the migrated seismic data in the inversion process. Algorithms from this class are assumed to have removed the band-limitation filter of the migrated real data $\left(\mathcal{S}=\mathcal{B}^{-1}\right)$ in the inversion process. Thus, equation 10 reduces to

$$
\hat{m}_{\text {inv }}(\mathbf{x})=\mathcal{A} \hat{m}(\mathbf{x}) .
$$

The second class of seismic inversion algorithms performs 1D inversion and hence will only partly remove the band-limitation filter from the migrated real data $\left(\mathcal{S}=\overline{\mathcal{B}}^{-1}\right)$. To simulate a $1 \mathrm{D}$ inversion image $\left(\hat{m}_{\text {inv }}^{1 \mathrm{D}}\right)$ that can be compared directly to the $1 \mathrm{D}$ inverted real data, the following relation is used:

$$
\hat{m}_{\text {inv }}^{1 \mathrm{D}}(\mathbf{x})=\overline{\mathcal{B}}^{-1} \mathcal{B}\{\mathcal{A} \hat{m}(\mathbf{x})\}
$$

This relation is rewritten by introducing an approximated angle filter $\mathcal{A}$ as

$$
\hat{m}_{\text {inv }}^{1 \mathrm{D}}(\mathbf{x})=\mathcal{A} \hat{m}(\mathbf{x}),
$$

where $\mathcal{A}=\mathcal{B}^{-1} \mathcal{B} \mathcal{A}$ (Figure 14).

\section{VALIDATION TEST: SIMULATING 1D INVERTED DATA}

The finite-difference data of the half-graben model are depth migrated using the correct velocity model. This process produces a focused migration image (not shown). Next, an impedance image is obtained from 1D inversion of the migrated data by running a commercial sparse-spike inversion algorithm. We followed the process of Ferguson and Margrave (1996) to compute an impedance image. This computation results in an impedance image we call the reference 1D inversion image (not shown).

To simulate this result, we could have used a simulated migration image as input to the inversion algorithm. On the other hand, inversion impedance data are simulated by performing a multidimensional spatial convolution between the approximated angle filter and the normal-incidence reflectivity model (equation 13), then using the result as input to the algorithm described by Ferguson and Margrave. The result is shown in Figure 15a.

The approximated angle filters at $x=1100 \mathrm{~m}, z=1100 \mathrm{~m}$ to $x$ $=1500 \mathrm{~m}, z=1500 \mathrm{~m}$ are computed by performing a $1 \mathrm{D}$ inversion of the spatial-resolution filters (Figure 15b). The corresponding inversion wavelet can be obtained by selecting the $k_{x}=0$ values of 


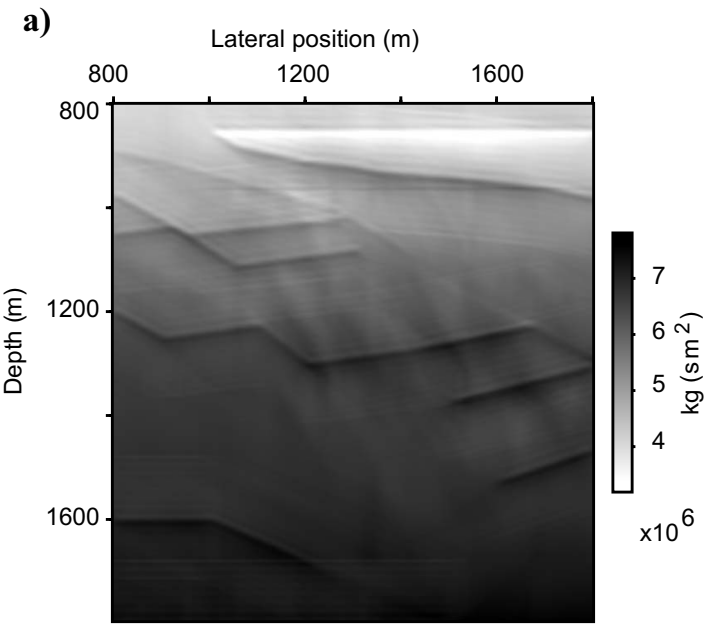

b) Lateral position $(m)$ Lateral position $(m)$ Lateral position $(m)$

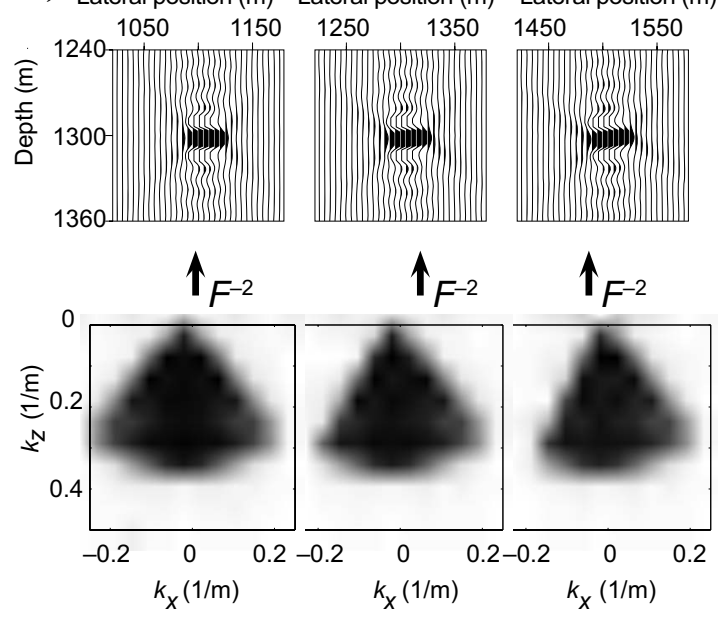

Figure 15. (a) A simulated impedance image of the target zone, obtained from 1D inversion, is simulated by convolving the approximated angle filters (b) with nine equally distributed areas in the model of Figure 13a. (b) Three of the nine approximated angle filters at a constant depth of $1300 \mathrm{~m}$. The spatial-resolution filters are shown in the space domain (above) and in the wavenumber domain (below).

the wavenumber-domain spatial-resolution filter. Alternatively, a different wavelet — for example, one with a rotated phase — can be used to show the effect on an inversion image.

A trace at $1150 \mathrm{~m}$ is selected from the reference, the simulated 1D inversion result, and the impedance model. The comparison indicates that the reference and simulated data show the same trend, which is smoother than the input impedance data (Figure 16a). Around the location of the steeply dipping faults ( $x$ is $1200-1800 \mathrm{~m}$ ), one depth slice at $1050 \mathrm{~m}$ is selected (Figure 16b). The reference and simulated 1D inversion images match each other nicely. However, they do not recover the trend of the input impedance because the influences of the low-frequency model and the suppression of specific dipping reflectors need to be restored. This shows that a simulated impedance image should include the effect of the limited recording aperture and propagation effects of the overburden. Current seismic inversion practices do not include these effects.
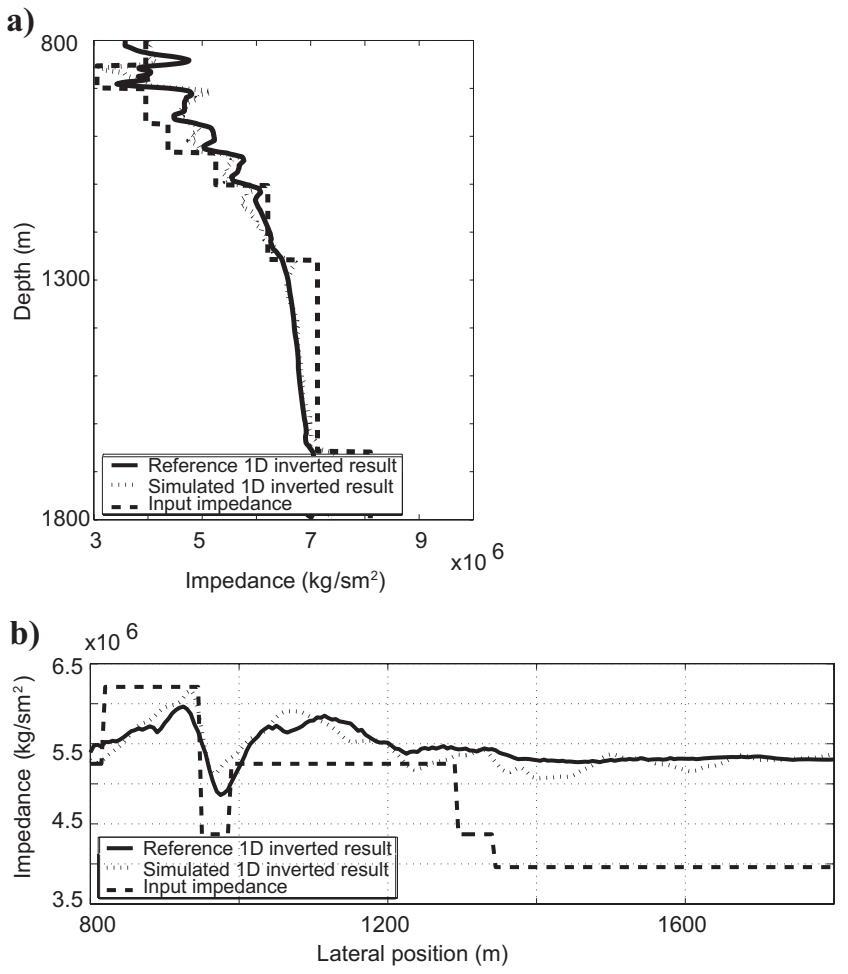

Figure 16. (a) A trace comparison at lateral position $1150 \mathrm{~m}$ of Figure $15 \mathrm{a}$, the reference and the impedance model. (b) A depth slice comparison at a depth of $z=1050 \mathrm{~m}$ of Figure 15a, the reference and the impedance model.

\section{CONCLUSIONS}

To iteratively test different geologic scenarios that differ only in a target zone, we propose to filter a model by spatial-resolution and angle filters to simulate migrated and inverted data, respectively.

The key is the spatial-resolution filter, the result of the combined operator $\mathrm{C}$, which combines the forward and the migration operators. It can be decomposed into an angle $\mathcal{A}$ and a band-limitation filter $\mathcal{B}$. This process can be summarized as $\mathcal{C}=\mathcal{B} \mathcal{A}$. The spatial-resolution filter captures all acquisition, seismic processing, and overburden-related effects, which cause seismic uncertainties. The simulated migrated data show the effects of vertical and horizontal smearing and exhibit a different wavelet stretch for different reflector dips, similar to migrated real data. Results of the real data example show that horizontal smearing, which is not simulated by the 1D convolution result, is essential to match the real migration result.

A condition for simulating migrated data to test geologic scenarios is that the macrovelocity model used to migrate the real data would approximate the macrovelocity model of the earth. If this condition is not met, a defocused spatial-resolution filter can be used to simulate the effects of the velocity error on the migrated data.

Seismic inversion aims to remove band-limitation from the migrated real data. We argue that $1 \mathrm{D}$ inversion of the migrated real data only partly removes the band-limitation filter. An approximated angle filter simulates inverted data for this specific class of inversion algorithms. The validation tests show that the simulated and reference 1D inversion impedance data can be compared directly. In particular, the simulated 1D inversion impedance data showed large phase and amplitude errors, especially for layers dipping more than 
approximately $25^{\circ}$, matching the corresponding observations from the reference data.

\section{ACKNOWLEDGMENTS}

StatoilHydro financially supported the research reported in this paper. This support is gratefully acknowledged. The permission of StatoilHydro to use and publish the real data is greatly appreciated. Susanne Lund Jensen and David Hunt are acknowledged for help with the real data example. The first author kindly thanks the Netherlands Organization for Scientific Research (NWO) for providing a travel grant. Fugro-Jason is acknowledged for free use of the software package GeoWorkBench. Finally, we thank reviewers Peeter Akerberg, Isabelle Lecomte, and two anonymous reviewers for many suggestions that improved this paper.

\section{REFERENCES}

Aki, K., and P. G. Richards, 2002, Quantitative seismology: University Science Books.

Berkhout, A. J., 1987, Applied seismic wave theory: Elsevier Science Publ. Co., Inc.

Beylkin, G., and R. Burridge, 1984, Linearized inverse scattering problems in acoustics and elasticity: Wave Motion, 12, 15-52.

Bleistein, N., J. K. Cohen, and J. W. Stockwell, Jr., 2000, Mathematics of multidimensional seismic imaging, migration, and inversion: SpringerVerlag.

Braaksma, H., J. N. Proust, J. A. M. Kenter, G. G. Drijkoningen, and N. Filippidou, 2006, Sedimentological, petrophysical, and seismic characterization of an Upper Jurassic shoreface-dominated shelf margin (the Boulonnais, northern France): Journal of Sedimentary Research, 76, 175-199.

Chen, J., and G. T. Schuster, 1999, Resolution limits of migrated images: Geophysics, 64, 1046-1053.

Claerbout, J. F., 1976, Fundamentals of geophysical data processing: McGraw-Hill Book Company.

Deregowski, S. M., and F. Rocca, 1981, Geometrical optics and wave theory of constant offset sections in layered media: Geophysical Prospecting, 29, 374-406.

Devaney, A. J., 1984, Geophysical diffraction tomography: Transactions in Geoscience and Remote Sensing, 22, 3-13.

Ferguson, R. J., and G. F. Margrave, 1996, A simple algorithm for band-limited impedance inversion: CREWES Research Report 8, 1-10.

Gelius, L. J., I. Lecomte, and H. Tabti, 2002, Analysis of the resolution function in seismic prestack depth imaging: Geophysical Prospecting, 50, $505-515$.

Gjøystdal, H., A. Drottning, I. Lecomte, and M. Branston, 2007, Advances in quantitative model-assisted seismic interpretation: First Break, 25, $95-102$.

Gjøystdal, H., E. Iversen, R. Laurain, I. Lecomte, V. Vinje, and K. Astebol, 2002 , Review of ray theory applications in modelling and imaging of seismic data: Studia Geophysica et Geodaetica, 46, 113-164.

Hill, S., B. Dragoset, and A. Weglein, 1999, An introduction to this special section: The new world of multiple attenuation: The Leading Edge, 18, 38

Jensen, S. L., D. Hunt, and S. A. Petersen, 2004, Seismic modeling of sulphate dissolution and karst collapse related deformation: 66th Annual Conference and Technical Exhibition, EAGE, Expanded Abstracts, C013.

Kallweit, R. S., and L. C. Wood, 1982, The limits of resolution of zero-phase wavelets: Geophysics, 47, 1035-1046.

Lancaster, S., and D. Whitcombe, 2000, Fast-track 'coloured' inversion: 70th
Annual International Meeting, SEG, Expanded Abstracts, 1572-1575.

Lecomte, I., 2006, Illumination, resolution, and incidence-angle in PSDM: A tutorial: 76th Annual International Meeting, SEG, Expanded Abstracts, $2544-2548$

Lecomte, I., and L. J. Gelius, 1998, Have a look at the resolution of prestack depth migration for any model, survey and wavefields: 68th Annual International Meeting, SEG, Expanded Abstracts, 1112-1115.

Lecomte, I., H. Gjøystdal, and Å Drottning, 2003, Simulated prestack local imaging: A robust and efficient interpretation tool to control illumination, resolution, and time-lapse properties of reservoirs: 73rd Annual International Meeting SEG, Expanded Abstracts, 1525-1528.

Loewenthal, D., L. Lu, R. Roberson, and J. Sherwood, 1976, The wave equation applied to migration: Geophysical Prospecting, 24, 380-399.

Matson, K., and B. Dragoset, 2005, An introduction to this special sectionMultiple attenuation: The Leading Edge, 24, 252.

Mittet, R., R. Sollie, and K. Hokstad, 1995, Prestack depth migration with compensation for absorption and dispersion: Geophysics, 60, 1485-1494.

Nemeth, T., C. Wu, and G. T. Schuster, 1999, Least-squares migration of incomplete reflection data: Geophysics, 64, 208-221.

Oldenburg, D. W., T. Scheuer, and S. Levy, 1983, Recovery of the acoustic impedance from reflection seismograms: Geophysics, 48, 1318-1337.

Petersen, S. A., 1992, A description of the geophysical technology and its impact on the construction of ideal information bases: 54th Annual Conference and Technical Exhibition, EAGE, Extended Abstracts, 74-75.

Petersen, S. A., B. A. Farrelly, and B. I. Braathen, 2003, Modeling of fluid effects in compartmentalized areas, an Oseberg South case study: 65th Annual Conference and Technical Exhibition, EAGE, Expanded Abstracts, A25.

Pratson, L., and W. Gouveia, 2002, Seismic simulations of experimental strata: AAPG Bulletin, 86, 129-144.

Sambridge, M., C. Begheim, F. J. Simons, and R. Snieder, 2006, How do we understand and visualize uncertainty?: The Leading Edge, 25, 542-546.

Santos, L. T., J. Schleicher, M. Tygel, and P. Hubral, 2000, Seismic modeling by demigration: Geophysics, $\mathbf{6 5}, 1281-1289$.

Schuster, G. T., and J. Hu, 2000, Green's function for migration: Continuous recording geometry: Geophysics, $\mathbf{6 5}, \mathbf{1 6 7 - 1 7 5}$.

Sheriff, R. E., 2001, Encyclopedic dictionary of applied geophysics: SEG.

Stolt, R. H., 1978, Migration by Fourier transform: Geophysics, 43, 23-48.

Thorbecke, J., K. Wapenaar, and G. Swinnen, 2004, Design of one-way wavefield extrapolation operators, using smooth functions in WLSQ optimization: Geophysics, 69, 1037-1045.

Thore, P., 2006, Accuracy and limitations in seismic modeling of reservoirs: 76th Annual International Meeting, SEG, Expanded Abstracts, $1674-1677$.

van Riel, P., and A. J. Berkhout, 1985, Resolution in seismic trace inversion by parameter estimation: Geophysics, 50, 1440-1455.

Veeken, P. C. H., and M. Da Silva, 2004, Seismic inversion methods and some of their constraints: First Break, 22, 47-70.

Vermeer, G. J. O., 2001, 3-D seismic survey design: SEG.

Verschuur, D. J., A. J. Berkhout, and C. P. A. Wapenaar, 1992, Adaptive surface-related multiple elimination: Geophysics, 57, 1166-1177.

Wapenaar, C. P. A., and A. J. Berkhout, 1989, Elastic wave field extrapolation, redatuming of single-and multi-component seismic data: Elsevier Science Publ. Co., Inc.

Wapenaar, K., A. J. Van Wijngaarden, W. Van Geloven, and T. Van der Leij, 1999, Apparent AVA effects of fine layering: Geophysics, 64, 1939-1948.

White, R., and R. Simm, 2003, Tutorial, Good practice in well tie: First Break, 21, 75-83.

Xie, X.-B., S. Jin., and R.-S. Wu, 2006, Wave-equation-based seismic illumination analysis: Geophysics, 71, no. 5, S169-S177.

Yilmaz, O., 2001, Seismic data analysis, in M. R. Cooper, ed., Seismic data analysis vol. 2: SEG 1001-2027.

Yu, J., J. Hu, G. T. Schuster, and R. Estill, 2006, Prestack migration deconvolution: Geophysics, 71, no. 2, S53-S62.

Zhu, J., L. Lines, and S. Gray, 1998, Smiles and frowns in migration/velocity analysis: Geophysics, 63, 1200-1209. 